\title{
The Great Matrix of All Beings
}

William Grassie

Metanexus Institute

\begin{abstract}
The Great Matrix of Being is ten hierarchical scales in nature-size, time, matter, energetics, electromagnetism, sound, information-ingenuity, sentience-consciousness, culturally constructed hierarchies, and the end product, emergent complexity. Some of these scales are empirically enumerated while the latter must be inductively inferred. These ten axes of the Great Matrix structure how matter, energy, and information combine to give rise to emergent complexity in a universe that otherwise marches toward entropic disorder. The Great Matrix is a useful mnemonic in the Big History curriculum for exploring causality, complexity, and important philosophical issues in contemporary science. This essay is an excerpt from a forthcoming book, Applied Big History: A Guide for Investors and Other Living Things.
\end{abstract}

Correspondence | William Grassie, william@grassie.net

Citation | Grassie, W. (2018) The Great Matrix of All Beings. Journal of Big History, II(2); 37 - 52.

DOI | http://dx.doi.org/10.22339/jbh.v2i2.2302

Learning "the big ideas of all the big disciplines," as famed investor Charlie Munger suggests, is not really all that hard. Indeed, he claims it is both fun and profitable. He recommends that we build "a latticework of mental modules."

Another word for lattice is matrix. A matrix is a structure, medium, or environment in which something develops. Matrices can be physical, biological, social, and mathematical. It can be a mold that shapes organisms, for instance, with intercellular matrices in our body. In mathematical usage, a matrix is like a spreadsheet - a grid of quantities and formulas, charts and comparisons, statistics and accounts, or more abstractly, geometric possibility spaces. The etymology of the word matrix is from the Latin for "womb" or "mother." We might also title this chapter "the Great Womb of All Beings."

In all of these senses, including perhaps especially the creative maternal, we begin by considering the Great Matrix as discovered and elucidated by science. I find this metaphor and mnemonic a useful way to navigate a lot of science in diverse disciplines, as well as to introduce important philosophical issues into the Big History curriculum.

Everything that exists in the universe, every process that science has discovered, every power of nature, all that constitutes our human bodies and brains, our histories and cultures - all this and more - can be located within a number of natural

1 Charles Munger, "Usc Law School Commencement Address, May 1, 2007," Genius.com, http://genius.com/Charliemunger-usc-law-commencement-speech-annotated. hierarchies and scales - size, time, matter, energetics, electromagnetism, sound, information-ingenuity, sentience-consciousness, cultural constructs, and emergent complexity. There are other scales and hierarchies, to be sure, but these ten natural scales are the warp and woof of the universe upon which all complexity is woven - is the first step to applying Big History to understanding "all the big ideas of all the big disciplines."

\section{1) Scales of Size}

It takes some effort to grok the scales of size from the macrocosm to the microcosm as discovered by science. ${ }^{2}$ How to internalize the new scales of science that extend far beyond our direct sense perceptions? In that sense, most of science is by definition counter-intuitive. You can't see much of what science is about-it's too large or too small, too fast or too slow. The existentialist in you may stay awake at night pondering how big the universe is, but it is perhaps more instructive to pay attention to how small entities can also be. The known universe is as many orders of magnitude smaller than us as it is larger. As physicist Richard Feynman observed, "there is a lot of room at the bottom."

The smallest unit in particle physics is the Planck

2 The word "grok" was a coined by Robert Heinlein in his 1961 science fiction novel Stanger in a Strange Land. The word is now listed in the Oxford English Dictionary, meaning "to understand intuitively or by empathy, to establish rapport with" and "to empathize or communicate sympathetically (with); also, to experience enjoyment." https://en.wikipedia.org/wiki/Grok 
scale $\left(1.62 \times 10^{-35} \mathrm{~m}\right)$. Beyond the Planck scale the concepts of size and distance break down and quantum indeterminacy becomes absolute-whatever that means. You will need to find a particle physicist to make more sense of these subatomic realms in which matter turns into miniscule fields and forces.

From the Plank scale we jump about 24 orders of magnitude in size pass the scale of subatomic particle to the scale of simple atoms. The diameter of a single hydrogen atom - one proton, one electron, and a lot of empty space-is approximately $0.1 \mathrm{~nm}$ (nanometers). Just to be clear, one nanometer $\left(10^{-9} \mathrm{~m}\right)$ is a billion times smaller than one meter.

Amino acids - small molecules essential to cell chemistry and varying in atomic composition - are approximately $0.8 \mathrm{~nm}$ in size.

DNA, the molecule deoxyribonucleic acid, is about 2 nanometers wide but varies dramatically in length. The longest human DNA is found in chromosome 1 and consists of 220 million base pairs. When stretched out it would be about 85 millimeters long and very, very thin. In terms of length, human DNA has some 3 billion base pairs, but that's nothing compared to the marbled lungfish, which clocks in at a 133 billion base pairs. At the other end of the DNA spectrum is the diminutive bacteria Carsonella ruddii with the smallest known genome containing 159,662 base pairs. DNA, as we will explore below, has an aperiodic property that allows it to encode information for cell construction and replication.

Prokaryotes - single-cell bacteria and archaea, more about that later - are the smallest and ur-unit of life. The smallest bacteria are about $150-250 \mathrm{~nm}$. The bacterium $E$. coli, part of your intestinal microbiome, measures up at approximately $2 \mu \mathrm{m}$ (micrometers or microns). Here we have moved up in the scale a thousand fold, from nanos to microns, from $10^{-9} \mathrm{~m}$ to $10^{-6} \mathrm{~m}$.

It takes imagination to grasp these orders of magnitude jumps in scale. At the other end of the size spectrum, we measure in light-years, which in spite of its name is a measurement of distance not time. One light-year is the distance that a photon travels in one Earth year-9.4 trillion kilometers in case you were wondering. The most distant thing that we know observationally in the universe is the background radiation from the big bang-13.8 billion light-years away from earth. 9.4 trillion kilometers times 13.8 billion is a really big number and an incomprehensible distance for mere humans to grasp - and yet we now do - though difficult to grok.

To get a feel for the relative size and scale of the universe, imagine holding a big beach ball one meter in diameter. It is summer and you're vacationing on Cape Cod, Massachusetts. If this beach ball were the size of the sun, then the next closest star, Proxima Centauri, would be another beach ball $6609 \mathrm{~km}$ away - the flight distance from Boston to Rome (the actual distance is 4.2 light years).

On the other hand, if your one-meter diameter beach ball were the size of our Milky Way galaxy, then the next closest galaxy - the Andromeda galaxy — would be another beach ball 25 meter away (the actual distance is 2.5 million light years). Indeed, at the scale of the galaxies, everywhere you looked on the beach that day, above, below, and all around, you would see beach ball galaxies in all directions - a 100 billion of them. In scale, galaxies are quite close to each other and gravitationally bound in a vast moving web, while the distance between stars within the galaxies is enormous and for the most part gravitationally not interactive with neighboring stars. When galaxies collide, as Andromeda and the Milky Way some day will, they pass through each other and nary a star will collide. The universe is a web of a hundred billion galaxies held together in a dynamic gravitational embrace.

The tendency is to focus on how puny we are in the scale of hundreds of billions of galaxies spanning billions of light years, but we should also remember how enormous we are when compared with the cells, molecules, atoms, and subatomic particles. Humans exist at scales measured in millimeters, centimeters, meters, and kilometers, which turn out to be about halfway between the very small and the very large. The human scale is also the only scale where certain types of complexity could exist. 
The Cosmic Uroboros is a graphic representation of the hierarchy of size. The image of a snake eating its own tail is a symbol appearing in ancient Egyptian, Greek, Norse, and Indian art. The symbol is thought to represent self-reflectivity and selfregeneration. In a rendering of the uroboros by cosmologist Joel Primack and co-author Nancy Abrams, the hierarchy of size is plotted in powers of ten along the circle. ${ }^{3}$ The universe is swallowing its own tail, because the very large scale of the universe loops back on the very small scale of subatomic particles. The particle accelerator at CERN recreates the intense energy of the early universe in order to study the constituents of matter. The sought-after "Grand Unified Theory" of fundamental physics (GUT in the image) would unite the four fundamental forces - gravity, electromagnetism, and the strong and weak atomic forces - connecting the subatomic and the cosmic dimensions, much as uroboros swallows its tail.

The first axis in the Great Matrix of all beings is size. Note again the curiosity and perhaps necessity that life exists halfway between the largest and smallest entities known to science. And take a moment also to cogitate and meditate on how your own being is built and functions bottom-up from small to ever larger combinatorials of atoms, molecules, and cells.

\section{2) Scales of Time}

The time scales of the universe are measured today in billions of years down to the nanosecond vibrations of cesium in atomic clocks. We have discovered a progressively true account of the 13.8-billion-year history of the universe, the 4.5-billion-year evolution of our planet, the 300,000 -year rise of our species, and the 10,000year accelerating drama of human civilization. This chronology is how Big History is generally approached in books and curricula, telling the story as an integrated narrative of complexity thresholds

3 Joel R. Primack and Nancy Ellen Abrams, The View from the Center of the Universe: Discovering Our Extraordinary Place in the Cosmos (New York: Riverhead, 2006). from the big bang to the 21 st century.

In brief, our omnicentric universe began some 13.8 billion years ago in a fantastically small, dense, hot, and symmetrical state. This universe expanded, cooled, and evolved into more differentiated and complex structures - forces, quarks, hydrogen, helium, galaxies, stars, heavier elements, complex chemistry, and planetary systems.

Some 4 billion years ago, in a small secondor third-generation solar system, the intricate biophysical processes of life began on at least one small planet circling a diminutive, slow burning star. Animate energy-matter on Earth was a marvelous new intensification of the creative dynamic at work in the universe. Life adapted, evolved, complexified. Fuel by the power of the sun, Earth became a jewel of a planet with ever more complex forms and relations giving rise to the wonders of the natural world.

Then some 7 million years ago, proto-humans emerged on the savanna of Africa. By a million years ago, they mastered fire and made simple tools. About 80,000 years ago, a small band of Homo sapiens wandered out of Africa. With their tools, fire mastery, language, and collective learning, our ancestors spread across the planet. We are the only large mammals to inhabit so many diverse bioregions.

For most of our prehistory, our common ancestors were hunter-gatherers, living in small tribes, probably not larger than 150 individuals. ${ }^{4}$ Our brains, instincts, emotions, and behavioral tendencies evolved to promote survival and reproduction in these social groups in diverse environments. Then 10,000 years ago, agriculture began, and with it growing populations of humans living in ever larger and more complex societies accumulating knowledge and knowhow, trading in genes, crops, animals, diseases, technologies, and ideas. The fossil fuel revolution began about 200 years ago and with it amazing growth in wealth and productivity. This unfolding leads us all the way to today, over 7

4 Robin Dunbar, Grooming, Gossip and the Evolution of Language (Cambridge: Harvard University Press, 1996). 
billion of us collectively transforming the planet and ourselves. The wonder of it all is that each of us is also a collection of transient atoms, recycled stardust become conscious beings, engaged in this global conversation brought to you by ephemeral electrons and photons cascading through the Internet and bouncing off of satellites.

That is Big History, in about 300 words and with a little poetic license. The story is generally understood in thresholds of emergent complexitythe early universe gives rise to stars and galaxies, the stars give rise to the periodic tables of elements and complex chemistry, the chemistry gives rise to simple bacteria, the bacteria give rise to eukaryotic cells, which complex cells give rise to the evolution of organisms, the plants and animals give rise to complex ecosystems with ever more sentient organisms, and sentience in our lineage gave rise humans consciousness, tool-making, language, fire mastery, collective learning, agriculture, and the modern revolution. Throughout the chronology, increased complexity is hierarchical, built from the bottom-up. Each threshold realizes new emergent properties that previously did not exist.

Einstein's theory of relativity, of course, links space and time in a continuum, so separating chronology and size scale is technically an artifact of our minds and not fundamental physics. Moreover, many of the equations of fundamental physics work forward or backward irrespective of time. And when we talk about the very fast, the very dense, and the very hot, the very cold, concepts of space and time can become elastic, but in between these extremes, time matters. For practical purposes, it is necessary to keep space and time separate. Together space and time provide the first two dimensions in the Great Matrix.

\section{3) Matter}

Technically, matter is solidified energy, as reflected in the iconic equation $\mathrm{E}=\mathrm{mc} 2$ - energy equals matter times the speed of light squared. However, just as with space and time, matter and energy may reliably be understood as distinct, at least in the context of the Great Matrix that matters most to us here on Earth.

Particle physicists advance our knowledge of smaller and smaller realms. Cosmologists advance our knowledge of large and larger realms. Neither has all the answers and their best working theoriesquantum mechanics and relativity - are mutually incompatible. The scale of atomic elements, however, is well established and central to all chemistry.

Matter turns out to be a scarce commodity in the universe. The universe is composed of about 70 percent cold dark energy. Another 25 percent consists of cold dark matter. Cosmologists don't really know what these are, ergo cold and dark, but infer their existence from the behavior of the web of galaxies in an expanding universe. Along with the cold dark energy and matter, there are invisible atoms. Hydrogen and helium, the atoms that fuel the stars, constitute about 0.5 percent of the universe. All the other visible atoms in the Periodic Table of Elements constitute less than 0.01 percent of the universe. Fortunately, we live in a chemically rich solar system on a very special planet. We are made of these remarkable and rare elements in this vast universe.

The character of elements is determined by the number of protons in their nucleus, along with isotopic variations in the number of neutrons and electrons bound to that nucleus. The discovery of periodicity and groupings of elements based on shared properties is one of the most remarkable achievements of science leading to all manner of new discoveries in physics and biology, not to mention engineering and technology. Chemistry may not have the same academic sex appeal as physics and biology, but it is the foundation upon which evolutionary and economic complexity builds.

\section{4) Scales of Energetics}

The intensity of energy flow is another axis in the Great Matrix. We can metaphorically equate energy with money. Energy does for nature what currencies do for human production and trade. And like all metaphors, it is literally not true. When Shakespeare calls time a beggar, he reminds us of our human 
finitude, but time is not literally a beggar. Metaphors are also important to science. Terms from computer science, for instance, are helpful in thinking about genetics, the neuroscience, and other fields.

Energetics is the currency of the Universal Central Bank, as guaranteed by the Laws of Thermodynamics. All of the regional banksastronomy, chemistry, biology, technology, economics, culture - can create their own energy currencies and markets because of this omnipotent guarantee. The Universal Central Bank does not offer fixed rates of return, but rather variable rates of losses in a predictable flux that impacts every domain at every scale. Energy dissipates. Entropy grows. That's not a problem; that's the singular opportunity.

The First Law of Thermodynamics observes that energy can be transferred and transformed, but not created or destroyed. At the first instance of the big bang, the universe received its lifetime allotment of energy-matter. That energy allowance is still present, just redistributed and less concentrated as the universe expands.

The Second Law of Thermodynamics states that in a closed system energy always moves for hot to cold, from order to disorder, from concentrations to entropy. What concerns us is not energy per se, but the flow of energy and the work that is done along the way.

Energy is the ability to transform a system-to do work. The Standard International unit of energy is the joule - defined as the energy required to lift a 1 kilogram object 1 meter vertically from the Earth's surface (i.e. against a gravitational force of 1 Newton). That energy is also released when that object falls back down. Lifting the object is mechanical work. The falling back down is kinetic energy - the release of potential energy in a gravitational field.

Physics distinguishes energy and power. Power is energy used over time. The Standard International unit of power is the Watt defined as 1 Joule per second. That 100 Watt light bulb is consuming 100 Joules per second, about the same amount of energy that your body is consuming, albeit in a different form.

Energy comes in different forms and typeselectromagnetic, chemical, thermal, kinetic, electrical, nuclear, gravitational - to name the major categories. There is no standard currency for energy in nature and society, though there are lots of standard equations for converting one energy form into the equivalent of another energy form.

For instance, we can convert the combustion of gasoline into the equivalent of human labor-11.6 days of labor to be precise. These calculations require multiple energy conversion, turning BTUs into kilowatts into 2500 food calories per day. We cannot, however, digest gasoline. It is a poison. The energy content of a single gallon of gasoline is equivalent to 28,977 food calories of energy or $51 \mathrm{Big} \mathrm{Mac}$ hamburgers to be precise. ${ }^{5}$

These kinds of conversions neglect important qualitative differences in energy types, but are nevertheless informative. Energy flow (i.e. power) is not the gasoline or the hamburgers - they are stored, potential chemical energy. Energy flow is the controlled burning of hydrocarbons by the machine or the body. A gallon of gasoline has a lot more potential chemical energy, than a gallon of you (which is mostly water), but the whole of you is able to harness the BTUs, Kilowatts, and Calories to amazing effects. We are the only entity that we know of that constructs complexity outside of our bodies in built devices using external energy sources. It goes all the way back to the controlled use of fire. The Prometheus myth got it right. Fire mastery is a godlike gift that separates us from all other animals.

The universe burns and humans have captured some of that energy. Physicists calculate the energy of the universe at the moment of the Big Bang as $10^{19} \mathrm{GeV}$ (billion electron volts). At the opposite end is absolute zero or minus 273.15 degrees Celsius (minus 459.67 degrees Fahrenheit).

All complex phenomena in the universe can be

5 One gallon of gasoline $=33.70 \mathrm{kWh}=28,977 \mathrm{kcal}$ 28,977 kcal@2500 kcal per day=food for 11.6 days of human labor

28,977 kcal=51 Big Macs@ @ 563 kcal per hamburger 
characterized by a flux of energy from hot to cold, from order to disorder, as required by the Second Law of Thermodynamics. Life excels at capturing energy to drive its creative processes.

One of the most counter-intuitive insights of modern science is the scale of energy density flow. Astronomer Eric Chaisson is credited with these calculations and insights. ${ }^{6} \mathrm{He}$ normalizes for the mass and time frame of each energetic system (ergs per second per gram). It turns out that a single-cell eukaryote has an energy density flow 500 times that of our sun. A photosynthesizing plant has about 5,000 times the energy density flow of the sun. A mammalian body has about 20,000 times the energy density flow of the sun. The human brain, consisting of about 2 percent of our body weight but consuming about 20 percent of our food energy, has an energy density flow about 75,000 times greater than the sun.

Of course, the sun is enormous and the flux of energy from the sun to the cold of outer space-3.86 x $10^{26}$ watts - far exceeds that of our human bodies running on the equivalent of a 100-watt light bulb. Remember that we are normalizing for the mass of the system and its time scale. What this means is that if you could expand the human body-ca. 70 kilograms - to the mass of the sun- $1.9 \times 10^{30}$ kilograms, then it would be 20,000 times more energetic (assuming you could feed it enough hamburgers).

And if we include all of the energy consumed outside of our bodies in our global civilization, then humans today achieve energy density flows average 250,000 times that of the sun. The privileged few of us who fly around the world - the energy richachieve energy density flows millions of times that of the sun. As we will further explore below, this exponential growth in energy density flow extends to the evolution of our technology. Humans and our

6 Eric Chaisson, Cosmic Evolution: The Rise of Complexity in Nature (Cambridge, MA: Harvard University Press, 2001); Epic of Evolution: Seven Ages of the Cosmos; "Energy Rate Density as a Complexity Metric and Evolutionary Driver," Complexity 16, no. 3 (2011); "Energy Rate Density. Ii. Probing Further a New Complexity Metric,” Complexity 17, no. 1 (2011). advanced machines may well achieve the greatest sustained energy density flows in the entire universe.

Remember that there are three ways to increase energy density flow: 1) you can consume more energy more efficiently, 2) you can compress the time scale of the system, and 3) you can decrease the mass of the system. With too little energy (e.g., starvation) the system will collapse. Too much energy flow and the system will also crash and burn. Each complex adaptive system has a range of optimal energy flows that are often limited within a narrow range, a kind of Goldilocks parameter.

There have been tremendous and accelerating increases in efficiency of our machines and technologies over the last 200 years. Much of this efficiency gain has been accomplished by reducing the mass of the system with new materials and technologies, for instance in the miniaturization that has occurred in electronics and computers, as well as more effective combustion processes with less heat wasted.

The increases in efficiency over the last hundred years are stunning. And yet overall consumption of energy continues to increase even as the technologies become more efficient. Our cars are more efficient, but more of us drive more miles. Our planes are more efficient, but more of us fly more miles. Our furnaces and air conditioners are more efficient, but we have bigger houses. These gains in efficiency mean increases in energy density flow. Energy efficiency is as important today as energy production, or for that matter labor productivity, because the reduced input can now be deployed elsewhere in creating additional economic growth and prosperity - more on that later. For now, we welcome energy density flow as the third axis in the Great Matrix.

The others scales below-light, sound, information-ingenuity, sentience-consciousness, evolved cultural hierarchies, and emergent complexity - are themselves forms of energy flow, a subset of energetics, each with unique properties essential to the evolution of complexity in nature and economics. 


\section{5) Scales of Electromagnetism}

Electromagnetism is one of the four fundamental forces in physics about which there is an active search for a Grand Unified Theory (GUT) that would combine these four forces in a single equation. In this exercise, we are going to ignore gravity and the two nuclear forces, because electromagnetism governs almost all of the phenomena that we encounter in daily life here on Earth.

Normal chemistry is all about the affinities of electrons and the indestructible elements of the periodic table. Negatively charged electrons are bound by electromagnetic attraction in orbitals around positively charged atomic nuclei that make up the periodic table of elements. Atoms combine into complex molecules through electromagnetic geometries and preferences. All chemistry, and therefore all biology, is governed by electromagnetic force. The ATP molecules in your cells, the neurons in your brain, the gasoline burning in your car, the food you eat, and all the electronic devices in your life-from the light bulb to the Internet - all utilize electromagnetic properties.

Electromagnetic radiation has three propertiesfrequency, wavelength, and photon-energy.

Frequency is measured in Hertz $(\mathrm{Hz})$ in a range of 104 to 1030 oscillations per second. Wavelength is measured in meters in a range of 10-12 to 106. Energy is measured in electronvolts $(\mathrm{eV})$ in a range of 106 to 10-15. The entire spectrum of electromagnetic radiation spans from long and slow oscillating radio waves at one end to short and fast oscillating gamma waves at the other end.

While the electromagnetic spectrum is a single, continuous physical phenomenon, our human eyes have evolved to perceive only a small range of visible light. We can feel infrared radiation on our skin, but the rest of the electromagnetic spectrum is invisible to our direct senses.

The entire spectrum is divided into ionizing and non-ionizing electromagnetic radiation (EMR). Short-fast EMR interacts energetically with atoms and molecules to break and remake chemical bonds. Long-slow EMR reacts minimally with most matter.
The break between ionizing and non-ionizing EMR has to do with the size of atoms and the wavelength of the photons. Ultraviolet EMR gives us sunburn. We use short-high frequency EMR to heat our food (microwave), look through our bodies (x-rays, magnetic imaging), and kill cells (Gamma ray).

Visible light is tucked in a sweet spot just below the range of the ionizing radiation. A prism famously splits white light into its constituent rainbow colors, each with their own specific range of wavelengths and frequency. Beyond purple we move into ultraviolet radiation ( $<380 \mathrm{~nm},<400$ terahertz). As the wavelengths get shorter, the frequencies get faster. Beyond red on our rainbow, we move into the infrared (>760 nm, >790 terahertz). The wavelengths get longer. The frequency slower.

Very high frequency (VHF) radio waves - used in television, radios, and other communication devices - range from 1 to 10 meters in wavelength and vibrate at a rate of 30 to 300 megahertz (MHz) (millions of oscillations per second). Electromagnetic radiation in the VHF band is long and interacts minimally with the atoms in the walls of your building or the cells in your body. It takes a special machine - your smartphone or radio - to tune into specific channels, to decode the broadcast woven in airwaves, and to convert that signal into sound or image for your enjoyment and edification.

Your eyes are basically a radio receiver at a different frequency. The photoreceptor cells in your eyes tune into specific ranges of electromagnetic radiation. Like the cells themselves, visible light is necessarily in the nanometer range in order to interact with nanometer-sized light-sensing organelles in the cells.

Electromagnetic radiation is central to all of the prosthetic "seeing" devices of science and technology - from radio telescopes to electron microscopes, from smartphones to global communications. These tools extend our vision, our abilities to see, hear, touch, taste, smell, and understand. What we know and know how to do goes far beyond our five natural senses. All of the prosthetic devices that extend human perception utilize the electromagnetic effect. 
All of the machines and motors that allow us to cross continents and move mountains utilize the electromagnetic effect.

Electromagnetic radiation, as recently harnessed by humans, is magical in how it has transformed our lives and our understanding of the universe. It is a continuous spectrum of wavelengths and frequencies, though divided into qualitatively different segments - radio waves, microwaves, infrared, visible light, ultraviolet, $\mathrm{x}$-ray, and gamma ray. The spectrum of electromagnetic radiation is the fourth axis in the Great Matrix of being - a form of energy flow and a critical subset of all energetics.

\section{6) Scales of Sound}

Sound is a vibration that propagates in a medium — gas, liquid, solid, or plasma. Unlike light, sound cannot travel through a vacuum; but like light, it has wavelength-frequency, directionality, intensity, and its own distinct speeds. Sound travels at the rate of 343 meters per second in dry air at $20 \mathrm{oC}$. Sound vibrations jostle molecules in a wave of kinetic energy, much like ripples on still water. Sound, like light, is also a subset of energetics.

An evolving capacity to sense pressure vibrations in the environment certainly has adaptive value for evolving organisms. Rudimentary "hearing" allowed organisms to receive directional information at a distance, thus improving survival and reproduction, while eventually leading also to forms of animal communications.

Curiously, the continuum of sound audible to humans ranges from $20 \mathrm{~Hz}$ to $20,000 \mathrm{~Hz}(17 \mathrm{~mm}$ to $17 \mathrm{~m}$ ), in contrast to the narrow range of visible light, which has no leaps in order of magnitude (790 terahertz to 400 terahertz- $760 \mathrm{~nm}$ to 380 $\mathrm{nm})$. Humans have much greater "depth" of sound perception than we have "depth" of light perception.

In any case, sound is a physical property of the universe, it has a hierarchical scale, and thus it provides yet another axis in the Great Matrix. Sound may not travel through outer space, but it is an essential component of evolution. And for humans, in particular, sound is central to our perception, communication, cooperation, enjoyment, survival, and reproduction.

\section{7) Hierarchies of Information-Ingenuity}

We might also postulate a scale of informationingenuity in the Great Matrix, even though we don't have a universal metric or even proper definition of information adequate to all scientific disciplines. Information - seemingly immaterial and ephemeral - can be a slippery metaphysical concept. In the broadest, most abstract sense, however, information is simply physical order in a universe that marches to the tune of disorder.

Out-of-equilibrium energetic systems spontaneously create physical order. ${ }^{7}$ This is how the weather works - a global system for dissipating the heat from the sun - most dramatically in hurricanes and tornados. The out-of-equilibrium physical order of the weather is information in the service of entropy. In gases and fluids, however, the spontaneous physical order is fleeting. It arises. It disappears. The weather is always changing. As Heraclitus observed 2500 years ago, you can't step into the same river twice.

Solid matter, however, can store information, at least for a time. Solid matter has memory. Its physical order can persist in ways that fluids and gas cannot. Solid matter, moreover, can also process that information to rearrange matter, energy, and information to make other stuff. In his book, Why Information Grows, Cesar Hidalgo calls the ability of matter to compute "one of the most amazing facts of the universe." (2477) Hidalgo writes:

\section{Information is not tangible; it is not a solid}

7 Ilya Prigogine was awarded the 1977 Nobel Prize in Chemistry "for his contributions to non-equilibrium thermodynamics, particularly the theory of dissipative structures." Ilya Prigogine and Gregoire Nicolas, eds., SelfOrganization in Nonequilibrium Systems: From Dissipative Structures to Order through Fluctuations (New York: Wiley, 1977); I. Prigogine and Isabelle Stengers, Order out of Chaos : Man's New Dialogue with Nature (New York, N.Y.: Bantam Books, 1984). 
or a fluid. It does not have its own particle either, but it is as physical as movement and temperature, which also do not have particles of their own. Information is incorporeal, but it is always physically embodied. Information is not a thing; rather, it is the arrangement of physical things. It is physical order, like what distinguishes different shuffles of a deck of cards.

(Hidalgo, 147)

If physical order is information, and if it takes energy to transform matter, and if it takes information to specify particular ordered states, then it takes energy to make and translate that information in, out, and back into matter. Encoding and reading information is thus also a subset of energetics, and thus governed by the Second Law of Thermodynamics. Information-ingenuity is not free, but it can be cheap. With information-ingenuity, evolution can minimize entropy and maximize creativity. Evolution offers the possibility of doing more with less through more efficiently coding.

DNA is a paradigmatic case of matter encoding and computing information. In his famous 1944 Dublin lectures and later book, What Is Life?, the physicist Edwin Schrödinger postulated that the code of life inside the cell needed to be an aperiodic crystal. Most crystals are periodic, meaning they form highly ordered microscopic structures. The molecules self-organize, based on their electromagnetic affinities, as they transition from liquid to solid in a tightly packed geometric lattice. These microscopic patterns then grow into macroscopic structures - diamonds, snowflakes, table salt, and metals of all kinds.

In an aperiodic crystal, however, variations in the molecular structure provide the possibility of coding information. Such is the molecule DNA - two strands of long molecules - polynucleotides - connected by variable bonds of adenine (A) to thymine (T) and cytosine $(\mathrm{C})$ to guanine $(\mathrm{G})$. The DNA molecule does not care about the actual order of these chemical bonds, only that $\mathrm{A}$ binds with $\mathrm{T}$ and $\mathrm{C}$ binds with $\mathrm{G}$ along the backbone of the two molecular strands.
The pattern of these base pairs - A-T and C-G bonds - is the template upon which DNA codes RNA and RNA codes proteins inside the cell. In cell division, the DNA replicates itself. Solid matter can encode information-ingenuity and then compute that information-ingenuity into living, reproducing, and evolving organisms.

In nature, DNA is always in solution inside the cytoplasm of cells. In a laboratory, however, biologists can separate and concentrate the DNA and then watch as the DNA molecules self-organize into crystalline forms. Because DNA is aperiodic, the crystals vary greatly, creating a profusion of psychedelic patterns under the microscope. (Image)

As scientists have developed techniques for manipulating DNA molecules, they have successfully used DNA to encode and decode digital information. It is slow and expensive work, but the potential is enormous. DNA can store orders of magnitude more data by volume than current computer hard drives with less energy and potentially over much longer time frames. A cubic millimeter of DNA can contain 5.5 petabits $\left(10^{15}\right)$. As of 2016, a single kilo of DNA would be sufficient to store all of the world's digital data. $^{8}$

In this technological feat - copying digital information in and out of DNA molecule - we encounter the dual nature of information that causes a lot of confusion. On the one hand, there is information as code. On the other hand, there is information as message. The code has no meaning in itself; it is merely an "arbitrary" vehicle for transmission. Information as message, however, is all about some meaning-construct this protein with this sequence of amino acids or interpret this string of A-T and C-G bonds as binary code for storing the digital contents of all computers. So we have two distinct definitions of information to keep separate, but that are also always connected.

In both senses - information as code and information as meaning - we might well imagine

8 Andy Extance, "How DNA Could Store All the World's Data," Nature, http://www.nature.com/news/how-dna-couldstore-all-the-world-s-data-1.20496. 
a scale in which information grows exponentially. Information measured as DNA base pairs, for instance, grows by orders of magnitude from prokaryotes to eukaryotes. Information grows again through multicellular organisms and with the evolution of the five senses - touch, taste, smell, sight, and sound - new codes conveying new meanings. And with the rise of symbolic language - spoken and later written - information as code and information as meaning experiences another exponential leap. All the while information is specifying and computing physical and social order, a continuous flux of creativity through our bodies, brains, and global civilization.

Ingenuity is simply information that does something creative, innovative, useful, and intelligent. The Latin root of the word "ingenuity" means "inborn," as in inborn genius. Indeed, the natural world is full of inborn genius. The inborn intelligibility-intelligence of nature is the precondition for scientific discovery and human technologies. We are surrounded by what philosopher Daniel Dennett refers to as "competence without comprehension." Most of our biological functions, including mental functions, occur without comprehension. Human consciousness and comprehension are recent development in evolutionary history and an ongoing story. We have only recently begun to comprehend our true place in the universe.

In computer science the smallest unit of information is a bit -0 or 1 . Technically, random garbage on your hard disk contains more "information" than your photos, music, documents, and applications. The latter are encoded in more compact algorithms. No such luck with random $1 \mathrm{~s}$ and $0 \mathrm{~s}$. By the information-as-code definition, disorder contains more "information" than order.

This seemingly esoteric discussion of information theory turns out to be central to economics and finance. The rise of complexity in nature and culture concerns us, not simply the amount of code required

9 Daniel C. Dennett, From Bacteria to Bach and Back (New York: W.W. Norton, 2017). to describe a particular state of physical order or disorder.

Humans are the most amazing computers of information-ingenuity, but we also encounter limits. Cesar Hidalgo introduces the concept of personbytes to represent the maximum amount of knowledge and know-how that an individual can acquire. Firmbytes are the maximum amount of knowledge and know-how that a firm can acquire. It takes time and effort to acquire expertise and skill. There is a limit to how much knowledge and knowhow one individual or one firm can acquire. And to accomplish complicated manufacturing or provide complicated services requires a lot of personbytes and firmbytes in collaboration. These limits are why complex economic activity requires networks of firms working in tandem with complex supply lines. Acquiring information-ingenuity is hard work, but it plays a central role in all disciplines and professions. Humans have taken the computing of energy, matter, and ingenuity to a whole new level.

So we imagine inductively another scale in the Great Matrix - a scale of information-ingenuitythe code and the meaning - that also grows exponentially. In evolution, it grows both as the quantity of DNA code, but also as the diversity of ingenious life forms. In human culture, it grows both as the quantity of code-spoken, printed, broadcast, and digital code - and as the quality of ingenuityknowledge and know-how that accumulate through collective learning across generations and across geographies.

\section{8) Hierarchies of Sentience-Consciousness}

We might postulate yet another axis in the Great Matrix: a hierarchy of sentience leading to consciousness. The membranes of microbes already have rudimentary sentience, in so far as they seek out food sources and flee harm. The immune system is also sentient, goal directed in response to its environment. The evolution of the central nervous system and the five senses further increased sentience. Animals evolve feeling and emotionsan amazing intensification of sentience. With the 
advent of symbolic language and collective learning, humans brought about another exponential leap in the scale of sentience-consciousness with intense first person subjectivity and social intersubjectivity. Free will is probably not the right term. Instead, we have constrained choice, but at an extent orders of magnitude beyond any of our animal kin.

Across the variety of animal species, brain-mind is an emergent phenomenon and potentially scalable. While there is no numeric scale for feeling and perception, sentience and consciousness, we can identify the ability of single cells to "sense" the presence of food or predators in the environment as perhaps the low end of the sentience spectrum in its most rudimentary form. A roundworm in a neuroscience lab has only a few hundred nerve cells, while a human brain has billions nerve cells. Surely, there are objective differences in bodybrain-mind complexity between round words and humans. Perhaps some day we will have such a scale throughout the animal kingdom.

Counting nerve cells alone, however, does not really give us an adequate measure of brainminds. Our human brain-minds require bodies and metabolism, vocal chords and oppositional thumbs, and an enriching social and natural environment, in order to realize their potentials.

While closely related to information-ingenuity, I treat sentience-consciousness as separate phenomena dealing primarily with the subjectivity, behavior, and agency of creatures. Human subjectivity and consciousness are on a kind of continuum throughout an evolutionary hierarchy. Sentience-consciousness is also a subset of energetics; and like our other scales in the Great Matrix, I imagine exponential leaps occurring throughout the drama of Big History.

Perhaps someday we may have a robust measure of sentience-consciousness that will allow us to compare dogs with cuttlefish, elephants with birds, and smart phones with smart people.

\section{9) Culturally Constructed Hierarchies}

Humans construct social and cultural hierarchies and scales that exist nowhere else in nature. These hierarchies have structured societies for good and ill throughout history. Some of these are evolved dispositions that we share with other animal species. Primate dominance hierarchy behavior may be deep in our genes, for instance, but the organizational flow charts at your company are not. All complex societies and institutions have social hierarchies reflected in their customs and laws, in their mating and childrearing practices, in and their occupations and role models. The alpha male - and sometimes alpha female - is such an archetype, one partially grounded in our evolved human nature, but also constructed by societies.

Applied mathematics also involves human constructed scales and hierarchies. While mathematics is also somehow discovered, humans have used these insights to construct new scales and hierarchies. In his famous 1960 paper physicist Eugene Wigner pondered "the unreasonable effectiveness of mathematics in the natural sciences." Indeed, mathematics is essential to understanding all of the scales and hierarchies of the Great Matrix. Mathematics has a miraculous ability to describe reality in new and profound ways. Mathematics, however, is not simply discovered "out there" in the universe; mathematics is also evolved and invented. And once mathematics is discovered-invented, it can become completely self-referential. It need not play with any reality except itself in a Neoplatonic universe of ideas.

A lot of science and economics is arithmeticmeasuring and counting. Complex modeling, however, requires the rocket scientists. And here, economic and scientific models begin to up run up against complexity and chaos theory. It is important to remember that the mathematics of finance and economics is not real. It need not refer to anything real in the way that the laws of physics do. The unit of measurement-currency-is a socially constructed fiction. No other species exchanges goods and services based on a symbolic system of value. Much like advanced mathematics, the measurements and models in economics and finance can be selfreferential with no basis in reality. Still, there is an 
"unreasonable effectiveness" of mathematics in our economic evolution. Indeed, mathematics first took hold in human brains with the growth of trade in early agricultural civilizations.

The accelerating drama of human evolution required us to create all kinds of social and cultural hierarchies, in order to scale human cooperation from intimate hunter-gatherer tribes to millions living in mega-cities around the world.

\section{0) Hierarchies of Emergent Complexity}

Taken together-size, time, matter, energy, and all the rest - bring us to the final scale in the Great Matrix of all beings - the scale of emergent complexity. Science has no numeric scale for complexity. Here too we need to appeal to informed intuition and induction, rather than some discreet, measurable qualia in nature. The emergence of complexity, however, is certainly one of key features of Big History with important implications for economics and finance. Emergence is a bottom-up process of energy, matter, and information creating top-down constraints on lower-level processes. Humans in general, and scientists in particular, are extreme examples the top-down capacities to constrain, collect, and transform energy, matter, and information in our built physical and cultural environments.

Big History traces eight or more thresholds of emergent complexity in the evolutionary narrative from the big bang to the 21 st century to create a pedagogically powerful narrative that enhances understanding, retention, excitement, and relevance - all badly needed in today's classrooms and society.

For instance, the creation of the heavy elements in the stellar foundries from which we derive the elements of the periodic table was a threshold of emergent complexity necessary for complex chemistry to later evolve. When complex chemistry catalyzed life, we saw again something new and different. And when the evolution of plants and animals gave rise to species with a central nervous system, complex brains, oppositional thumbs, vocal chords, symbolic language, tool-making, fire mastery, and collective learning, something new emerged again in the universe, at least on one small planet.

It is important to emphasize that emergent complexity requires lower levels of complexity to exist and function. Higher orders of complexity are built bottom-up, though emergent properties cannot be fully explained from below. With thresholds of emergent complexity, the Great Matrix is not simply a coordinate system of reality, but now also an epic narrative of becoming.

We can distinguish between four different kinds of emergent complexity in the new epic narrative of evolution:

1) Evolutionary Emergence: The grand arc of Big History is the story of the universe, Earth, and humanity. In the beginning, there were no stars, no galaxies, no periodic table of elements, no planets, no water, no complex chemistry, no life, and no intelligence. These emerged in a sequencethresholds of emergent complexity-over a timescale measured in billions and millions of years.

\section{2) Developmental Emergence: Each of us} begins our journey as a single cell in our mother's womb. Over nine months, that cell replicates and differentiates into a few hundred tissue-types of our bodies. The development continues through childhood, into adult bodies composed of some 40 trillion cells. In this example, developmental emergence occurs in a timescale measured in months, years, and decades.

3) Functional Emergence: Protons, neutrons, and electrons form atoms; atoms join together to form molecules; molecules attach to form more complex chemistry. The inner life of a cell is a dense and intense collection of millions of molecular machines undergoing trillions of mind-boggling chemical cascades per second. The atoms and the molecules are not alive, but when functioning together, they can form a living cell, organ, and organism. Functional emergence in our bodies occurs in a timescale 
measured in nanoseconds and hours.

4) Intelligence Emergence: With the advent of symbolic language and collective learning, we see the accelerating emergence of intelligence in humans. Science, technology, engineering, math, and medicine are the most obvious examples, as new insights and discoveries are built upon the successes of the past in a progressive and self-transcending learning process. This kind of emergence also occurs in the arts and humanities. The emergence of intelligence is a complex distributed phenomenon and is evolutionary (over the course of human history), developmental (over the course of an individual's lifetime), and functional (necessary for the workings of increasingly complex societies). ${ }^{10}$

While evolutionary emergence is the grand narrative of Big History, coming to recognize and understand developmental emergence, functional emergence, and intelligence emergence is no less important. The emergence of complexity is an ongoing process.

To truly appreciate Big History, we must not only count in billions of light-years, but also the amazing nanoscale functions and developments manifested by quadrillions of atoms dancing in and out of our bodies. We must also appreciate the history of knowledge, creativity, and discovery that characterizes the recent, dramatic, and accelerating successes of our species.

With these different concepts of emergence in hand, we can now begin to understand how the academic disciplines and departments of the modern university map onto the structure of the universe itself. From the bottom up, we begin with fundamental theory, particle physics, nuclear physics, solid-state physics, and chemistry. The disciplines then split into inanimate and animate tracks. Inanimate matter goes from physical chemistry to material sciences, earth sciences, space sciences,

$10 \mathrm{I}$ am in debt to George Ellis for many of these insights. See George F.R. Ellis, How Can Physics Underlie the Mind? Top-Down Causation in the Human Context (New York: Springer, 2016). astronomy, and cosmology. Cosmology, it should be noted, also takes us back to fundamental theory and particle physics, so the top and bottom are connected in the disciplines of physics and astronomy. Life, animate matter, leads to the many divisions of biology all the way to the human sciences, and indeed also all the humanities. Throughout our review of specialization and division of labor in universities, we also encounter applied sciences - for instance, engineering, medicine, and economics. The distinction between science and applied science is not easy to maintain.

\section{A Multi-Dimensional Matrix}

The ten dimensions of the Great Matrix give us ten ways to measure reality-by time, size, matter, energetics, electromagnetism, sound, information-ingenuity, sentience-consciousness, cultural constructs, and the end product, emergent complexity. Some of these are inductive scales that cannot be measured in numeric units. We might measure information, as bits for instance, but we cannot enumerate the ingenuity embedded in the code. We don't have a consciousness meter that we can put on your head. And beyond the crude measure of energy-density-flow, we don't have a scale for emergent complexity. It is a triumph in intellectual history that we have come to understand the scales of time, size, matter, and the varieties energetics. These are reliably real and profoundly practical insights about how our universe and our economy function.

All phenomena, all academic disciplines, and all economic activities can be located in reference to these hierarchical scales. It is this Great Matrix of bottom-up and top-down causality that allows complexity to grow.

Medieval Europeans once understood the universe to be a Great Chain of Being. All the entities of he world - animal, vegetable, mineral — were hierarchically organized. At the bottom were metals, precious metals, and precious stones. Then came plants and trees, followed by wild animals and domesticated animals. Humans were also hierarchically ordered from children to women 
to men and further into the different ranks of commoners, nobility, princes, and kings. The Great Chain of Being continued up into the celestial realm - moon, stars, angels, and archangels - to the very top where God presides over the entire creation. This scala naturae provided humans with a natural order, which they also understood to be a natural human order that structured their societies.

Science, or so the story goes, disrupted this view of the universe and ourselves. Copernicus, Galileo, and Kepler broke the crystalline spheres of Ptolemy and demoted Earth from the center of the universe to an insignificant periphery. Darwin understood plants and animals, including the human animal, to be evolving from common ancestors all the way back into the proverbial primordial slime. Freud showed that rational man was really an unconscious mess and hardly aware of, let alone in control of, his own thoughts and passions.

The Great Chain of Being was rendered a tangled web of happenstance in an enormous universe devoid of transcendence and meaning. God was rendered an unnecessary or incompetent creator. The new existentialists and Stoics argued that the universe was indifferent, that humans were insignificant, that our consciousness was epiphenomenal, and that our evolution merely accidental. Note simply that while there is no Great Chain of Being as the medieval Europeans understood, there is a great deal of order in the universe as discovered by contemporary science. There is a Great Matrix to which all beings belong that extends far beyond our direct senses and perception.

Humans are not at the top of the scales of size and time, but somewhere in the middle. On the energetics, information-ingenuity, sentienceconsciousness, and emergent complexity scales, however, humans are off the chart. The human niche is particularly favored in the matrix for the time being - each of us a nexus of causal relationships (physical, biological, social, economic, psychological, mental), realizing extraordinary flows of energy and ingenuity, intensities of subjective experience, and accelerating transformations in the modern period.

In our drive toward specialization and division of labors, we rarely reflect on these natural hierarchies and what they might mean for our understanding of science, self, and society. "The ongoing fragmentation of knowledge and resulting chaos are not reflections of the real world but artifacts of scholarship," writes Harvard biologists E.O. Wilson in his book, Consilience: The Unity of Knowledge. ${ }^{11}$ Vartan Gregorian, president of the Carnegie Corporation, similarly observes:

The fundamental problem underlying the disjointed
curriculum is the fragmentation of knowledge
itself. Higher education has atomized knowledge
by dividing it into disciplines, subdisciplines,
and sub-subdisciplines - breaking it up into
smaller and smaller unconnected fragments of
academic specialization, even as the world looks
to colleges for help in integrating and synthesizing
the exponential increases in information brought
about by technological advances. The trend has
serious ramifications. Understanding the nature of
knowledge, its unity, its varieties, its limitations,
and its uses and abuses is necessary for the success
of democracy... We must reform higher education to
reconstruct the unity and value of knowledge. ${ }^{12}$
Understanding how the Great Matrix actually Understanding how the Great Matrix actually
works on different scales is an exercise in zooming in, zooming out, and changing perspectives. In the process, we better understand the emergent complexity from physics to chemistry, from cell biology to human brains, from individual producerconsumers to global markets. We need to take account of the energy, matter, and ingenuity that flows through nature and our economy.

We are never outside the bio-social-physical matrix, but in this scientific and philosophical exercise we seem to stand away, looking down on the matrix from above. As far as we know, no other entity in the universe has achieved this capacity, and it is in this domain that humans are

11 Edward O. Wilson, Consilience: The Unity of Knowledge (New York: Knopf, 1998).

12 Vartan Gregorian, "Colleges Must Reconstruct the Unity of Knowledge," Chronicle of Higher Education 50, no. 39 (2004). 
no longer middling creatures of the matrix. Our self-transcendence, realized especially through the progress in science, economics, art, and culture, is a super and completely natural emergent phenomenon. We come to understand the matrix from the inside out, though the matrix knows nothing of us.

It is awe-inspiring to grok any or all of these natural scales and hierarchies. Simply appreciating the scales of size and time is awesome. Try also to analyze the flows of energy embodied in the objects and activities in your immediate environment. A process analysis of energy in our built environment gives us a new understanding of the economic world. Training your eyes to "see" the energy flows embedded in the world around you is sure to wake you up in the morning. I look at my library and recall that a ton of paper embodies on average about 35 gigajoules (GJ). This is about as much energy as is needed to manufacture a ton of good-quality steel, to say nothing of the human labor that went into writing these books. I walk through my home and recollect that the average three-bedroom, wood-framed house in North America embodies about 500 GJ of energy in its construction, which is equivalent to 4,157 gallons of gasoline (or something like 48,183 days of human labor). I get into my car and consider that a midsized passenger car requires about 110 GJ to build and might consume about 50 GJ of fuel annually. Over ten years, the energy cost of that car would total about $680 \mathrm{GJ}$-more than that of the average three-bedroom house mentioned above.

It doesn't stop there. If your eyes could detect the radio waves around you, the empty space of your room would be filled with an altogether different kind of rainbow - the chaotic patterns of dozens of overlapping VHF broadcasts would be evident all around you. If your skin could feel these electromagnetic waves, your entire body would be vibrating and tingling at different frequencies. Indeed, some of the airwaves entering through the window right now have traveled 13.8 billion years from the background radiation of the big bang to be with you today. Or if you prefer the wave function of quantum phenomena, consider yourself connected across space and time in a very immediate sense to the distant reaches of the universe. Understanding the Great Matrix provides a kind of instant mystical experience in which you can deconstruct and reconstruct your sense of self and world within these multidimensional scales and hierarchies.

Science offers a sixth sense - a way of seeing beyond the walls of Plato's cave into the realm of what is real. It isn't rocket science to sort-of-know how things actually work, how the pieces form an entire puzzle. Of course, god and the devil are both in the details, but it sure helps to have the picture on the puzzle box when trying to fit the pieces together.

\section{Bibliography}

Berry, Thomas, and Brian Swimme. Universe Story, The: From the Primordial Flaring Forth to the Ecozoic Era. San Francisco, CA: Harper, 1992.

Chaisson, Eric. Cosmic Evolution: The Rise of Complexity in Nature. Cambridge, MA: Harvard University Press, 2001.

—. "Energy Rate Density as a Complexity Metric and Evolutionary Driver." Complexity 16, no. 3 (2011): 27-40.

_. "Energy Rate Density. Ii. Probing Further a New Complexity Metric." Complexity 17, no. 1 (2011): 44-63.

. Epic of Evolution: Seven Ages of the Cosmos. New York: Columbia University Press, 2006.

Christian, David. Maps of Time: An Introduction to Big History. Berkeley: University of California Press, 2004.

Dennett, Daniel C. From Bacteria to Bach and

Back. New York: W.W. Norton, 2017.

Dunbar, Robin. Grooming, Gossip and the

Evolution of Language. Cambridge: Harvard

University Press, 1996.

Ellis, George F.R. How Can Physics Underlie the Mind? Top-Down Causation in the Human Context. New York: Springer, 2016.

Extance, Andy. "How DNA Could Store All the World's Data." Nature, http://www.nature.com/news/ 
how-dna-could-store-all-the-world-s-data-1.20496.

Grassie, William J. Reinventing Nature: Science Narratives as Myths for an Endangered Planet. Philadelphia: doctoral dissertation, Temple University Department of Religion, 1994. Gregorian, Vartan. "Colleges Must Reconstruct the Unity of Knowledge." Chronicle of Higher Education 50, no. 39 (6/4/2004 2004): B12.

Hidalgo, Cesar. Why Information Grows: The Evolution of Order, from Atoms to Economies. New York: Basic Books, 2015.

Munger, Charles. "Usc Law School

Commencement Address, May 1, 2007." Genius. com, http://genius.com/Charlie-munger-usc-lawcommencement-speech-annotated.

Prigogine, I., and Isabelle Stengers. Order out of Chaos: Man's New Dialogue with Nature. New York, N.Y.: Bantam Books, 1984.

Prigogine, Ilya, and Gregoire Nicolas, eds. SelfOrganization in Nonequilibrium Systems: From Dissipative Structures to Order through Fluctuations. New York: Wiley, 1977.

Primack, Joel R., and Nancy Ellen Abrams. The View from the Center of the Universe: Discovering Our Extraordinary Place in the Cosmos. New York:

Riverhead, 2006.

Smil, Vaclav. Energy in Nature and Society. Cambridge, MA: MIT Press, 2007.

Spier, Fred. Big History and the Future of Humanity. New York: Wiley-Blackwell, 2011. - The Structure of Big History: From the

Big Bang until Today. Amsterdam: Amsterdam University Press, 1996.

Weschler, Lawrence. Waves Passing in the Night: Walter Murch in the Land of the Astrophysicists New York: Bloomsbury, 2017.

Wilson, Edward O. Consilience: The Unity of Knowledge. New York: Knopf, 1998. 